\title{
Double the Prevalence of Stage 2 Hypertension Readings in a Small Group of American Pre- clinical Medical Students Compared to Young Adults Diagnosed with Stage 2 Hypertension in the United States
}

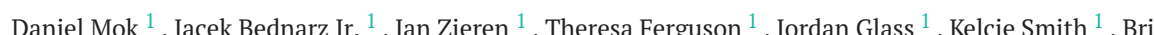
Yonish ${ }^{1}$

1. Family Medicine, Lincoln Memorial University - DeBusk College of Osteopathic Medicine, Harrogate, USA

Corresponding author: Daniel Mok, daniel.mok@lmunet.edu

\section{Abstract \\ Objective}

The aim of this study is to compare the prevalence of hypertension (HTN) in pre-clinical (first- and secondyear) medical students at Lincoln Memorial University to that of the United States population and identify risk factors in this group.

\section{Materials and Methods}

Students from the DeBusk College of Osteopathic Medicine completed a survey that queried age, gender, tobacco use, alcohol consumption, diet, aerobic exercise, mental health, social support, amount of sleep per night, and past medical history. Omron BP710N (Omron 3 series) sphygmomanometers were used to measure blood pressures in the left arm. Waist circumference was measured around the umbilicus. HTN stages were defined under the 2017 ACC/AHA guidelines. Univariate, binominal, and multinomial regression analyses of risk factors were performed using SPSS v22.0 with $\alpha=0.05$.

\section{Results}

Of the 213 students surveyed, $49.8 \%$ (106/213) were males, $49.3 \%$ (105/213) were females, and $0.9 \%(2 / 213)$ declined to reveal their gender. The mean age of the sample was 25.8 years $(\mathrm{SD}=2.75$ years) and the range was between 21 and 37 years. Under the 2017 ACC/AHA guidelines, 36.6\% (78/213) were normotensive; $16.4 \%$ (35/213) had elevated blood pressure; 29.1\% (62/213) had stage $1 \mathrm{HTN}$; and 17.8\% (38/213) had stage 2 HTN. A multinomial logistic regression model was significant, $\chi^{2}(9)=82.934, p<0.001$, explained $34.9 \%$ (Nagelkerke $\mathrm{R}^{2}$ ) of the variance in $\mathrm{HTN}$, and correctly classified $50.2 \%$ of cases. In comparison to

Received 02/19/2020

Review began $03 / 06 / 2020$ Review ended 03/19/2020 Published 03/28/2020

\section{(c) Copyright 2020}

Mok et al. This is an open access article distributed under the terms of the Creative Commons Attribution License CC-BY 4.0., which permits unrestricted use, distribution, and reproduction in any medium, provided the original author and source are credited. normotensive females, normotensive males are 2.81 times more likely (95\% CI: $1.04-7.61 ; p=0.042$ ) to develop stage $2 \mathrm{HTN}$; increasing waist circumference by $1 \mathrm{~cm}$ in normotensive students was associated with a 10\% increase (95\% CI: 1.06-1.15; $p<0.001$ ) in developing stage $2 \mathrm{HTN}$; and sleeping < 6 hours per night was associated with 4.33 times increased (95\% CI: 1.52-12.34; $p=0.006$ ) likelihood of developing stage 2 HTN with respect to normotensive students who sleep for 6-8 hours a night.

\section{Conclusion}

Our sample of medical students has a 2.4 times higher prevalence of stage $2 \mathrm{HTN}$ readings in comparison to adults aged 18-39 according to the 2015-2016 national CDC hypertension prevalence report. Risk factors including male gender and sleeping less than 6 hours per night are significant predictors of elevated and stage 2 HTN. Waist circumference is predictive of stage 1 HTN and stage 2 HTN. Additional studies should be conducted to increase the sample size in order to better assess the prevalence of stage 2 HTN in American medical students.

Categories: Cardiology, Internal Medicine, Preventive Medicine

Keywords: sleep, hypertension, medical, students, american, waist circumference

\section{Introduction}

In a 2015-2016 study, The Centers of Disease Control and Prevention (CDC) estimates that $7.5 \%$ of the US adults of age 18-39 years have high blood pressure, which they defined as a systolic pressure greater than or equal to $140 \mathrm{~mm} \mathrm{Hg}$ or diastolic pressure greater than or equal to $90 \mathrm{~mm} \mathrm{Hg}$ [1]. As of today, the aforementioned blood pressure criteria meet the definition for stage 2 HTN under the relatively new 2017 American College of Cardiology (ACC)/American Heart Association (AHA) guidelines. These are the first changes to hypertension guidelines since 2003, which have lowered the threshold in defining hypertension. 
Hypertension now encompasses elevated, stage $1 \mathrm{HTN}$, stage $2 \mathrm{HTN}$, and hypertensive crisis. Individuals who met the stage 1 HTN criteria before 2017 are now classified as having stage 2 HTN based on the 2017 guidelines. In addition, the category of prehypertension has been eliminated and replaced with either elevated or stage $1 \mathrm{HTN}$. Normal is defined as a systolic pressure less than $120 \mathrm{~mm} \mathrm{Hg}$ and diastolic pressure less than $80 \mathrm{~mm} \mathrm{Hg}$; elevated is a systolic pressure of 120-129 $\mathrm{mm} \mathrm{Hg}$ and diastolic pressure less than $80 \mathrm{~mm} \mathrm{Hg}$; stage $1 \mathrm{HTN}$ is a systolic pressure of $130-139 \mathrm{~mm} \mathrm{Hg}$ and/or diastolic pressure of $80-89 \mathrm{~mm}$ $\mathrm{Hg}$; stage $2 \mathrm{HTN}$ is a systolic pressure of $140 \mathrm{~mm} \mathrm{Hg}$ and greater and/or a diastolic pressure of $90 \mathrm{~mm} \mathrm{Hg}$ and greater; a hypertensive crisis is defined as a systolic pressure greater than $180 \mathrm{~mm} \mathrm{Hg}$ and/or a diastolic pressure greater than $120 \mathrm{~mm} \mathrm{Hg}$. Due to these changes in the guidelines, it is now estimated that $46 \%$ of the US population has hypertension [2].

Research on HTN involving American medical students is lacking. This is one of the earliest known studies to survey blood pressures of American medical students and classify the readings under the 2017 ACC/AHA guidelines in an attempt to understand the prevalence of HTN in this population. It is important to understand this population's blood pressure, as first- and second-year medical students are at risk to acquire modifiable risk factors due to the nature of the education. Students are sedentary for most of the day, experience significant amounts of stress, and lack time to exercise and to cook healthy meals. There have been studies that looked at the blood pressure in the African, Middle Eastern, and South-East Asian medical students [3-5]. These studies have found high blood pressure among students; however, reported prevalence varies wildly among studies.

It is well known that many individuals are unaware of having HTN due to the lack of symptoms from the disorder, and that HTN in young adults is a predisposition for developing cardiovascular disease [6]. Early identification is crucial because a physician can help a patient modify behaviors in order to stop the progression of the disorder. Hypertension independently increases the risk of cardiovascular disease even after adjusting for multiple cardiovascular risk factors such as total cholesterol, smoking status, body mass index (BMI), and diabetes mellitus [6]. Therefore, because HTN goes unnoticed and HTN independently elevates risk for cardiovascular disease, it is important for young adults with modifiable risk factors to monitor their blood pressure and make changes to their behavior.

It seems that HTN may be a problem in medical students; therefore, this study's goal will be to compare the prevalence of HTN readings in pre-clinical (first and second year) medical students at the Lincoln Memorial University to that of the US population and identify risk factors in this group.

\section{Materials And Methods Design and ethical considerations}

First- and second-year medical students were surveyed during the 2018-2019 academic year. The population of the first- and second-year students enrolled at the Lincoln Memorial University was 460 students. In order to achieve a confidence level of $95 \%$ with a $5 \%$ margin of error and a $50 \%$ population proportion for this population, a target sample size of 210 students was necessary according to Cochran's sample size formula.

The Institutional Review Board at Lincoln Memorial University approved this study, protocol number: 756 V.0. The aim of this study and protocol to obtain physical measurements were explained to students. Written consent was obtained from each participant. Students were reassured that participation was in no way mandatory and students had the right to withdraw from the study at any time with no consequences.

\section{Setting}

The study was conducted in Harrogate, Tennessee at Lincoln Memorial University - DeBusk College of Osteopathic Medicine.

\section{Sample}

First- and second-year medical students were recruited to participate after lectures, tutorials, and teambased learning exercises. Eligibility criteria included being a first- or second-year medical student over the age of 18. Exclusion criteria included tobacco use 30 minutes prior to blood pressure measurement and any physical exercise 30 minutes prior to blood pressure measurement. Caffeine intake was not considered an exclusionary criterion for this study. Regular caffeine intake does increase blood pressure in the long term [7]. However, individuals who chronically drink caffeinated beverages do not experience acute spikes in blood pressure after ingestion [8].

\section{Data collection}

Participants were asked to fill out a paper questionnaire prior to having their blood pressure and waist circumference measured (Figure 1). The questionnaire queried age; gender; tobacco use; alcohol use; consumption of soft drinks, fast food, high glycemic foods, and red meats; aerobic exercise; mental health; social support; amount of sleep per night; history of diabetes; history of hypertension; and family history. To identify individuals at risk for major depressive disorder (MDD), we asked participants if they have felt down, 
depressed, or hopeless and if they have little interest or pleasure in doing things over the last two weeks. Answering yes to both these two questions has a sensitivity of $83 \%$ and specificity of $92 \%$ for detecting MDD [9]. To identify individuals at risk for generalized anxiety disorder (GAD), we asked participants if they have been feeling nervous anxious or on edge and if they have been unable to control or stop worrying for the past 2 weeks [10]. Answering yes to both the two previous questions would deem the participant at risk for GAD.

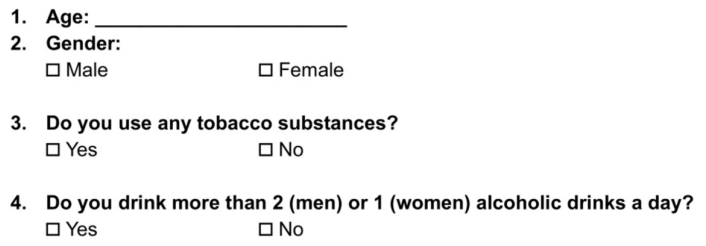

5. Do you consume any of the following items one or more times in an average week?

$\begin{array}{lcc}\text { Do you consume any of the following items one or more times in an average } & \square \text { Yes } & \square \text { No } \\ \text { Soft Drinks } & \square \text { Yes } & \square N o \\ \text { Fast Food } & \square \text { Yes } & \square N o \\ \text { High Glycemic (e.g. Chips, Cake, Chocolate Bars) } & \square \text { Yes } & \square \text { No }\end{array}$

6. Do you engage in aerobic activity (such as brisk walking) three to four times a week averaging 40 minutes per session?

$\square$ Yes

$\square$ No

7. On average how many hours of sleep do you get each night. $\square$ Less than 6 hours $\quad 6-8$ hours $\square$ More than 8 hours

8. Over the last 2 weeks, have you felt down, depressed or hopeless? $\square$ Yes $\square$ No

9. Over the last 2 weeks, have you had little interest or pleasures in doing things? पYes $\mathrm{No}$

10. Over the past 2 weeks have you been feeling nervous, anxious, or on edge? $\square$ Yes $\square$ No

11. Over the past 2 weeks have you been unable to control or stop worrying? $\square$ Yes $\quad$ No

12. While school is in session, do you have a good social support system? $\square$ Yes $\square$ No

13. Do you have any family history of the following? Cardiovascular Event $\square$ Yes $\square$ No Type 2 Diabetes $\quad \square$ Yes $\quad \square$ No

14. Have you been diagnosed with any of the following? Hypertension $\square$ Yes $\square$ No Type 2 Diabetes $\square$ YNo

15. If you answered yes to any items in Question 14, are you taking medication for this? पYes $\square$ No $\quad$ N /A

16. Average Blood Pressure Measurement:

17. Waist Circumference (centimeters):

FIGURE 1: Blank sample questionnaire

Blood pressure was measured by six trained researchers using Omron BP710N sphygmomanometers. Researchers practiced taking blood pressures prior to surveying students in order to ensure measurement accuracy. In order to minimize the effects of white coat hypertension, we used automated blood pressure cuffs to reduce clinician-participant anxiety and we took multiple readings separated in time. Blood pressures were measured according to the AHA's proper seated office blood pressure measurement guidelines with some modifications [11]. Participants were asked to relax and sit in a chair with their feet flat on the floor and back supported. Prior to measuring a participant's blood pressure, a paper questionnaire was given to the participant to fill out. If participants had additional queries with respect to any question on the questionnaire, participants were told; that no additional information could be provided and that they could either answer as they had interpreted the question or skip the question altogether. Blood pressure readings were taken when the participant had been seated for equal to or more than 3 minutes. Researchers were instructed that neither they nor the participant was to talk while the machine was taking a measurement. Participants' left arms were supported via a table that was at the height of their heart during measurement. After the first measurement was conducted, participants were asked to rest for 1-2 minutes 
before a second blood pressure reading was taken. A third measurement was taken 1-2 minutes after the second blood pressure reading if the difference in systolic or diastolic pressure between the first and second readings exceeded $10 \mathrm{~mm} \mathrm{Hg}$. The average of the two or three blood measurements was recorded for the individual.

A majority of the procedural methodologies in this study emulated that of the 2015-2016 CDC study to ensure that the data of this study would be comparable to that of the CDC [1]. Measuring blood pressure at only one point in time, inclusion/exclusion criteria, and measurement protocol were replicated. It was noted in the CDC's procedural methodology that measurements were obtained during a single examination visit. In this study, 2-3 blood pressure measurements were also taken during one encounter. In the CDC's determination of HTN prevalence, the exclusion criteria included those younger than 18 years of age, pregnant, had rashes, gauze dressings, casts, edema, paralysis, tubes, open sores or wounds, withered arms, a-v shunts, or radical mastectomy.

Waist circumference was measured with a tape measure wrapped above the iliac crests around the level of the umbilicus. Measurements were done directly on the skin; however, if the student requested, a measurement was taken over a T-shirt. Participants were asked to inhale and exhale after the tape measure was wrapped snug around the waist. Measurement was taken after the participant finished exhaling [12]. Measurements were recorded to the nearest centimeter.

\section{Statistical analysis}

The data were analyzed using SPSS v22.0 to tally the frequency of the risk factors and blood pressure categories. A binomial logistic regression analysis was used to test significant risk factors for hypertension. Hypertension and normotension were the nominal variables and the independent risk factors were waist circumference, age, gender, tobacco use, alcohol use, soft drink consumption, fast food consumption, high glycemic food consumption, red meat consumption, aerobic exercise, feeling depressed, anhedonia, anxiety, uncontrolled worrying, major depressive disorder, generalized anxiety disorder, and social support. A multinomial logistic regression was also used where the nominal variables were normotension, elevated, stage $1 \mathrm{HTN}$, and stage $2 \mathrm{HTN}$ and the independent risk factors were the significant risk factors obtained from the binomial regression analysis. A Chi-square test was used to assess the difference in the proportions of risk factors in corresponding blood pressure categories and genders. Independent t-tests were used to compare the mean waist circumference, systolic pressure, and diastolic pressure with respect to gender.

\section{Results}

\section{Description of sample}

The sample was made up of 213 students of which $49.8 \%$ (106/213) were males, $49.3 \%(105 / 213)$ were females, and $0.9 \%(2 / 213)$ declined to reveal their gender. The mean age of the sample was 25.8 years $(\mathrm{SD}=$ 2.75 years) and the range was 21-37 years. Under the 2017 ACC/AHA guidelines $36.6 \%$ (78/213) were normotensive; $16.4 \%(35 / 213)$ had elevated blood pressure; $29.1 \%(62 / 213)$ had stage $1 \mathrm{HTN}$; and $17.8 \%$ (38/213) had stage 2 HTN. Only $2.3 \%$ (5/212) of students have a known diagnosis of hypertension and $0.5 \%$ $(1 / 212)$ have a known diagnosis of type 2 diabetes mellitus. There were 11 surveys out of the 213 which had at least one unanswered question. Therefore, 202 complete surveys were used in the binomial and multinomial regression analysis. In univariate analysis, all answered questions from the 213 surveys were used.

\section{ACC/AHA hypertension levels \& associated risk factors}

Univariate analysis of modifiable and nonmodifiable risk factors in normotensive, elevated, stage 1 HTN and stage 2 HTN categories showed statistically significant differences in waist circumference $\left(X^{2}(12, N=213)=\right.$ 52.227, $p<0.001)$; gender $\left(X^{2}(3, N=211)=30.091, p<0.001\right)$; tobacco use $\left(X^{2}(3, N=211)=8.798, p=0.032\right)$; fast food consumption $\left(X^{2}(3, N=210)=7.882, p=0.049\right)$; and amount of sleep per night $\left(X^{2}(6, N=212)=\right.$ $19.355, p=0.004)$. (Table 1 )

\begin{tabular}{|c|c|c|c|c|c|c|c|c|c|c|c|c|}
\hline & \multicolumn{2}{|c|}{ Normotensive } & \multicolumn{2}{|c|}{ Elevated } & \multicolumn{2}{|c|}{ Stage 1} & \multicolumn{2}{|c|}{ Stage 2} & \multirow{2}{*}{ Test Statistic } & \multirow{2}{*}{$\mathbf{P}$} & \multicolumn{2}{|l|}{ Total } \\
\hline & Count & $\%$ & Count & $\%$ & Count & $\%$ & Count & $\%$ & & & Count & $\%$ \\
\hline \multicolumn{13}{|c|}{ Waist Circumference (cm) } \\
\hline $60-69$ & 16 & 20.5 & 2 & 5.7 & 5 & 8.1 & 2 & 5.3 & \multirow{4}{*}{$52.227^{\star}$} & \multirow{4}{*}{$<0.001^{\star}$} & 25 & 11.7 \\
\hline 70-79 & 30 & 38.5 & 12 & 34.3 & 13 & 21 & 1 & 2.6 & & & 56 & 26.3 \\
\hline 80-89 & 18 & 23.1 & 16 & 45.7 & 19 & 30.6 & 10 & 26.3 & & & 63 & 29.6 \\
\hline 90-99 & 11 & 14.1 & 4 & 11.4 & 16 & 25.8 & 14 & 36.8 & & & 45 & 21.1 \\
\hline
\end{tabular}




\begin{tabular}{|c|c|c|c|c|c|c|c|c|c|c|c|c|}
\hline$\geq 100$ & 3 & 3.8 & 1 & 2.9 & 9 & 14.5 & 11 & 28.9 & & & 24 & 11.3 \\
\hline \multicolumn{13}{|l|}{ Age } \\
\hline $21-23$ & 15 & 19.2 & 5 & 14.3 & 12 & 19.4 & 6 & 15.8 & \multirow{6}{*}{13.654} & \multirow{6}{*}{0.552} & 38 & 17.8 \\
\hline $24-26$ & 41 & 52.6 & 21 & 60 & 31 & 50 & 17 & 44.7 & & & 110 & 51.6 \\
\hline $27-29$ & 17 & 21.8 & 7 & 20 & 13 & 21 & 9 & 23.7 & & & 46 & 21.6 \\
\hline $30-31$ & 2 & 2.6 & 1 & 2.9 & 3 & 4.8 & 3 & 7.9 & & & 9 & 4.2 \\
\hline $32-33$ & 1 & 1.3 & 1 & 2.9 & 3 & 4.8 & 0 & 0 & & & 5 & 2.3 \\
\hline$>33$ & 2 & 2.6 & 0 & 0 & 0 & 0 & 3 & 7.9 & & & 5 & 2.3 \\
\hline \multicolumn{13}{|l|}{ Gender } \\
\hline Male & 21 & 27.3 & 26 & 74.3 & 34 & 55.7 & 26 & 68.4 & \multirow{2}{*}{$30.091^{*}$} & \multirow{2}{*}{$<0.001^{*}$} & 107 & 50.7 \\
\hline Female & 56 & 72.7 & 9 & 25.7 & 27 & 44.3 & 12 & 31.6 & & & 104 & 49.3 \\
\hline \multicolumn{13}{|c|}{ Tobacco Use } \\
\hline Yes & 1 & 1.3 & 1 & 2.9 & 7 & 11.5 & 1 & 2.6 & \multirow{2}{*}{$8.798^{\star}$} & \multirow{2}{*}{$0.032^{\star}$} & 10 & 4.7 \\
\hline No & 76 & 98.7 & 34 & 97.1 & 54 & 88.5 & 37 & 97.4 & & & 201 & 95.3 \\
\hline \multicolumn{13}{|c|}{ Alcohol Use } \\
\hline Yes & 0 & 0 & 1 & 2.9 & 4 & 6.7 & 2 & 5.3 & \multirow{2}{*}{5.246} & \multirow{2}{*}{0.155} & 7 & 3.3 \\
\hline No & 78 & 100 & 34 & 97.1 & 56 & 93.3 & 36 & 94.7 & & & 204 & 96.7 \\
\hline \multicolumn{13}{|c|}{ Soft Drinks } \\
\hline Yes & 27 & 34.6 & 13 & 37.1 & 30 & 49.2 & 18 & 48.6 & \multirow{2}{*}{4.048} & \multirow{2}{*}{0.256} & 88 & 41.7 \\
\hline No & 51 & 65.4 & 22 & 62.9 & 31 & 50.8 & 19 & 51.4 & & & 123 & 58.3 \\
\hline \multicolumn{13}{|c|}{ Fast Food } \\
\hline Yes & 33 & 42.3 & 18 & 51.4 & 30 & 50 & 26 & 70.3 & \multirow{2}{*}{$7.882^{*}$} & \multirow{2}{*}{$0.049^{*}$} & 107 & 51 \\
\hline No & 45 & 57.7 & 17 & 48.6 & 30 & 50 & 11 & 29.7 & & & 103 & 49 \\
\hline \multicolumn{13}{|c|}{ High Glycemic } \\
\hline Yes & 50 & 64.1 & 22 & 62.9 & 34 & 56.7 & 29 & 76.3 & \multirow{2}{*}{3.921} & \multirow{2}{*}{0.27} & 135 & 64 \\
\hline No & 28 & 35.9 & 13 & 37.1 & 26 & 43.3 & 9 & 23.7 & & & 76 & 36 \\
\hline \multicolumn{13}{|c|}{ Red Meats } \\
\hline Yes & 35 & 44.9 & 23 & 65.7 & 35 & 58.3 & 24 & 63.2 & \multirow{2}{*}{6.142} & & 117 & 55.5 \\
\hline No & 43 & 55.1 & 12 & 34.3 & 25 & 41.7 & 14 & 36.8 & & 0.105 & 94 & 44.5 \\
\hline Aerobic & ercise & & & & & & & & & & & \\
\hline Yes & 36 & 46.8 & 18 & 52.9 & 32 & 52.5 & 17 & 44.7 & & & 103 & 49 \\
\hline No & 41 & 53.2 & 16 & 47.1 & 29 & 47.5 & 21 & 55.3 & . & & 107 & 51 \\
\hline Amount & Sleep & & & & & & & & & & & \\
\hline$<6$ & 11 & 14.1 & 13 & 37.1 & 15 & 24.6 & 15 & 39.5 & & & 54 & 25.5 \\
\hline 6 to 8 & 60 & 76.9 & 20 & 57.1 & 46 & 75.4 & 23 & 60.5 & $19.355^{\star}$ & $0.004^{\star}$ & 149 & 70.3 \\
\hline$>8$ & 7 & 9 & 2 & 5.7 & 0 & 0 & 0 & 0 & & & 9 & 4.2 \\
\hline Major De & essiv & & & & & & & & & & & \\
\hline Yes & 10 & 12.8 & 4 & 11.4 & 10 & 16.4 & 9 & 23.7 & & & 33 & 15.6 \\
\hline No & 68 & 87.2 & 31 & 88.6 & 51 & 83.6 & 29 & 76.3 & 2.84 & 0.417 & 179 & 84.4 \\
\hline
\end{tabular}




\section{Cureus}

\begin{tabular}{|c|c|c|c|c|c|c|c|c|c|c|c|c|}
\hline Yes & 19 & 24.4 & 7 & 20 & 14 & 23 & 11 & 28.9 & \multirow{2}{*}{0.858} & \multirow{2}{*}{0.836} & 51 & 24.1 \\
\hline No & 59 & 75.6 & 28 & 80 & 47 & 77 & 27 & 71.1 & & & 161 & 75.9 \\
\hline \multicolumn{13}{|c|}{ Loss of Pleasure } \\
\hline Yes & 14 & 17.9 & 5 & 14.3 & 10 & 16.4 & 12 & 31.6 & \multirow{2}{*}{4.658} & \multirow{2}{*}{0.199} & 41 & 19.3 \\
\hline No & 64 & 82.1 & 30 & 85.7 & 51 & 83.6 & 26 & 68.4 & & & 171 & 80.7 \\
\hline \multicolumn{13}{|c|}{ Generalized Anxiety Disorder } \\
\hline Yes & 16 & 20.5 & 8 & 22.9 & 15 & 24.6 & 6 & 15.8 & \multirow{2}{*}{1.164} & \multirow{2}{*}{0.762} & 45 & 21.2 \\
\hline No & 62 & 79.5 & 27 & 77.1 & 46 & 75.4 & 32 & 84.2 & & & 167 & 78.8 \\
\hline \multicolumn{13}{|c|}{ Uncontrolled Anxiety } \\
\hline Yes & 50 & 64.1 & 26 & 74.3 & 38 & 62.3 & 21 & 55.3 & \multirow{2}{*}{2.923} & \multirow{2}{*}{0.404} & 135 & 63.7 \\
\hline No & 28 & 35.9 & 9 & 25.7 & 23 & 37.7 & 17 & 44.7 & & & 77 & 36.3 \\
\hline \multicolumn{13}{|c|}{ Worrying } \\
\hline Yes & 18 & 23.1 & 8 & 22.9 & 16 & 26.2 & 6 & 15.8 & \multirow{2}{*}{1.476} & \multirow{2}{*}{0.688} & 48 & 22.6 \\
\hline No & 60 & 76.9 & 27 & 77.1 & 45 & 73.8 & 32 & 84.2 & & & 164 & 77.4 \\
\hline \multicolumn{13}{|c|}{ Social Support } \\
\hline Yes & 73 & 93.6 & 33 & 94.3 & 57 & 93.4 & 35 & 92.1 & \multirow{2}{*}{0.153} & \multirow{2}{*}{0.985} & 198 & 93.4 \\
\hline No & 5 & 6.4 & 2 & 5.7 & 4 & 6.6 & 3 & 7.9 & & & 14 & 6.6 \\
\hline \multicolumn{13}{|c|}{ Family History: Cardiovascular Event } \\
\hline Yes & 41 & 52.6 & 17 & 48.6 & 27 & 45 & 19 & 50 & \multirow{2}{*}{0.791} & \multirow{2}{*}{0.852} & 104 & 49.3 \\
\hline No & 37 & 47.4 & 18 & 51.4 & 33 & 55 & 19 & 50 & & & 107 & 50.7 \\
\hline \multicolumn{13}{|c|}{ Family History: Type 2 Diabetes } \\
\hline Yes & 32 & 41 & 17 & 48.6 & 23 & 38.3 & 16 & 42.1 & \multirow{2}{*}{0.977} & \multirow{2}{*}{0.807} & 88 & 41.7 \\
\hline No & 46 & 59 & 18 & 51.4 & 37 & 61.7 & 22 & 57.9 & & & 123 & 58.3 \\
\hline
\end{tabular}

\section{TABLE 1: Distribution of each risk factor in its corresponding blood pressure category}

Blood pressure readings $(n=213)$ were taken from students where 11 students did not answer at least one survey question. Pearson's chi-square was performed for each risk factor. ${ }^{*} p<0.05$

In the binomial logistic regression model where the dichotomous outcome variable was hypertension in reference to normotension, the significant predictive risk factors were found to be waist circumference (OR: 1.04; $95 \%$ CI: $1.004-1.081 ; p=0.028$ ); male gender (OR: $1.351 ; 95 \%$ CI: $1.662-8.963 ; p=0.002$ ); and sleeping under 6 hours (OR: 3.547 ; 95\% CI: $1.511-8.328 ; p=0.004$; Table 2 ). 


\section{Cureus}

\begin{tabular}{|c|c|c|c|c|c|}
\hline \multirow{2}{*}{ Risk Factors } & \multirow{2}{*}{ B } & \multirow{2}{*}{ Significance } & \multirow{2}{*}{ Odds Ratio } & \multicolumn{2}{|c|}{$95 \%$ Confidence Interval } \\
\hline & & & & Lower & Upper \\
\hline Waist Circumference & 0.041 & $0.028^{*}$ & 1.04 & 1.004 & 1.081 \\
\hline Age & -0.061 & 0.376 & 0.94 & 0.823 & 1.077 \\
\hline Male Gender & 1.351 & $0.002^{\star}$ & 3.86 & 1.662 & 8.963 \\
\hline Tobacco Use & 1.251 & 0.333 & 3.5 & 0.278 & 43.969 \\
\hline Alcohol Use & 21.626 & 0.999 & $2.5 \times 10^{\wedge} 2$ & 0 & $\dagger$ \\
\hline Soft Drinks & 0.443 & 0.269 & 1.56 & 0.71 & 3.414 \\
\hline Fast Food & -0.074 & 0.854 & 0.93 & 0.423 & 2.04 \\
\hline High Glycemic & -0.026 & 0.949 & 0.98 & 0.445 & 2.136 \\
\hline Red Meats & 0.441 & 0.264 & 1.56 & 0.717 & 3.369 \\
\hline Aerobic Exercise & 0.001 & 0.997 & 1 & 0.461 & 2.176 \\
\hline$<6$ hours of sleep & 1.266 & $0.004^{*}$ & 3.55 & 1.511 & 8.328 \\
\hline Major Depressive Disorder & 1.487 & 0.203 & 4.43 & 0.448 & 43.748 \\
\hline Feeling Depressed & -0.425 & 0.502 & 0.65 & 0.19 & 2.257 \\
\hline Loss of Pleasure & -1.223 & 0.181 & 0.29 & 0.049 & 1.766 \\
\hline Generalized Anxiety Disorder & 1.268 & 0.442 & 3.56 & 0.14 & 90.167 \\
\hline Uncontrolled Anxiety & -0.103 & 0.806 & 0.9 & 0.397 & 2.049 \\
\hline Worrying & -0.801 & 0.605 & 0.45 & 0.022 & 9.312 \\
\hline Social Support & -0.407 & 0.607 & 0.67 & 0.141 & 3.14 \\
\hline Family History of Cardiovascular Event & -0.261 & 0.498 & 0.77 & 0.362 & 1.638 \\
\hline Family History of Type 2 Diabetes & 0.253 & 0.554 & 1.29 & 0.556 & 2.982 \\
\hline
\end{tabular}

TABLE 2: Binomial regression of the risk factors of hypertension (encompassing elevated, stage $1 \mathrm{HTN}$, and stage 2 HTN) compared to normotension

Omnibus chi-square $=61.572, P<0.001$; Nagelkerke $R^{2}: 0.359$. The model predicts $74.8 \%$ of cases correctly $(n=202) . B$ is the coefficient for the respective risk factor in the regression. ${ }^{*} p<0.05+$ SPSS could not calculate an upper $\mathrm{Cl}$ interval.

Cl, confidence interval

Further multinomial logistic regression analysis of the significant predictors from the binomial regression showed that male gender is a significant predictor of elevated (OR: $11.50 ; 95 \% \mathrm{CI}: 3.92-33.80 ; \mathrm{p}<0.001$ ) and stage 2 HTN (OR: $2.81 ; 95 \%$ CI: 1.04-7.61; $p=0.042)$. Sleeping less than 6 hours per night is also a significant predictor of elevated (OR: 4.96 ; $95 \%$ CI: $1.77-13.92 ; p=0.002$ ) and stage 2 HTN (OR: 4.33 ; 95\% CI: 1.52 $12.34 ; p=0.006)$. Increasing waist circumference by $1 \mathrm{~cm}$ is predictive of stage $1 \mathrm{HTN}(\mathrm{OR}: 1.05 ; 95 \% \mathrm{CI}$ : $1.02-1.09 ; p=0.006$ ) and stage 2 HTN (OR: 1.10 ; 95\% CI: 1.06-1.15; $p<0.001$ ) for this sample (Table 3). 


\section{Cureus}

\begin{tabular}{|c|c|c|c|c|c|}
\hline & \multirow{2}{*}{ B } & \multirow{2}{*}{ Significance } & \multirow{2}{*}{ Odds Ratio } & \multicolumn{2}{|c|}{$95 \%$ Confidence Interval } \\
\hline & & & & Lower & Upper \\
\hline \multicolumn{6}{|l|}{ Elevated Blood Pressure } \\
\hline Waist Circumference & -0.023 & 0.371 & 0.98 & 0.93 & 1.03 \\
\hline Male Gender & 2.443 & $<0.001^{*}$ & 11.5 & 3.92 & 33.8 \\
\hline$<6$ hours of sleep & 1.602 & $0.002^{*}$ & 4.96 & 1.77 & 13.92 \\
\hline \multicolumn{6}{|l|}{ Stage 1 Hypertension } \\
\hline Waist Circumference & 0.052 & $0.006^{*}$ & 1.05 & 1.02 & 1.09 \\
\hline Male Gender & 0.8 & 0.051 & 2.23 & 1 & 4.96 \\
\hline$<6$ hours of sleep & 0.786 & 0.092 & 2.19 & 0.88 & 5.48 \\
\hline \multicolumn{6}{|l|}{ Stage 2 Hypertension } \\
\hline Waist Circumference & 0.098 & $<0.001^{*}$ & 1.1 & 1.06 & 1.15 \\
\hline Male Gender & 1.034 & $0.042^{*}$ & 2.81 & 1.04 & 7.61 \\
\hline$<6$ hours of sleep & 1.466 & $0.006^{*}$ & 4.33 & 1.52 & 12.34 \\
\hline
\end{tabular}

TABLE 3: Multinomial regression of predictive risk factors of elevated, stage $1 \mathrm{HTN}$, and stage 2 HTN compared to normotension.

Likelihood ratio chi-square test $=82.934 ; p<0.001$; Nagelkerke $R^{2}: 0.349$. The model predicts $50.2 \%$ of cases correctly $(n=202)$. $B$ is the coefficient for the respective risk factor in the regression. ${ }^{*} p<0.05$.

\section{Differences in gender}

In this study, males made up a larger proportion of students with elevated, stage 1 HTN and stage 2 HTN, while females were the larger portion of normotensive students (Table 1). The mean abdominal circumference for the 106 males $(\mathrm{M}=89.72 \mathrm{~cm}, \mathrm{SD}=11.77)$ compared to the 105 females $(\mathrm{M}=78.89 \mathrm{~cm}, \mathrm{SD}=$ 11.14) was significantly larger, $\mathrm{t}(209)=6.860, p<0.001$. The systolic pressure for the 106 males $(\mathrm{M}=129.11$ $\mathrm{mmHg}, \mathrm{SD}=12.43)$ compared to the 105 females $(\mathrm{M}=115.95, \mathrm{SD}=11.73)$ was significantly larger, $\mathrm{t}(209)=$ $7.909, p<0.001$. However, the diastolic pressure for the 106 males $(\mathrm{M}=80.04 \mathrm{mmHg}, \mathrm{SD}=8.66)$ compared to the 105 females $(\mathrm{M}=78.51, \mathrm{SD}=9.16$ ) showed no statistically significant difference, $\mathrm{t}(209)=1.242, p=0.216$. Univariate analysis showed statistically significant differences in the blood pressure $\left(X^{2}(3, N=211)=\right.$ $28.295, p<0.001)$; waist circumference $\left(X^{2}(4, N=211)=47.509, p<0.001\right)$; soft drink consumption $\left(X^{2}(1, N\right.$ $=210)=5.007, p=0.025)$; fast food consumption $\left(X^{2}(1, N=209)=7.291, p=0.007\right)$; aerobic exercise engagement $\left(X^{2}(1, N=209)=5.216, p=0.022\right)$; generalized anxiety $\operatorname{disorder}\left(X^{2}(1, N=211)=6.538, p=\right.$ $0.011)$; worrying $\left(\mathrm{X}^{2}(1, N=211)=7.102, p=0.008\right)$; family history of a cardiovascular event $\left(X^{2}(1, N=210)=\right.$ $6.172, p=0.013)$; and family history of type 2 diabetes $\left(\mathrm{X}^{2}(1, N=210)=5.007, p=0.025\right)$ between males and females (Table 4).

\begin{tabular}{|c|c|c|c|c|c|c|c|c|}
\hline & \multicolumn{2}{|l|}{ Male } & \multicolumn{2}{|l|}{ Female } & \multirow{2}{*}{ Test Statistic } & \multirow{2}{*}{$\mathbf{P}$} & \multicolumn{2}{|c|}{ Male and Female Combinec } \\
\hline & Count & $\%$ & Count & $\%$ & & & Count & $\%$ \\
\hline \multicolumn{9}{|l|}{ Blood Pressure } \\
\hline Normotensive & 21 & 19.8 & 56 & 53.3 & \multirow{4}{*}{$28.295^{\star}$} & \multirow{4}{*}{$<0.001^{\star}$} & 77 & 36.5 \\
\hline Elevated & 25 & 23.6 & 10 & 9.5 & & & 35 & 16.6 \\
\hline Stage 1 Hypertension & 34 & 32.1 & 27 & 25.7 & & & 61 & 28.9 \\
\hline Stage 2 Hypertension & 26 & 24.5 & 12 & 11.4 & & & 38 & 18 \\
\hline
\end{tabular}




\section{Cureus}

\begin{tabular}{|c|c|c|c|c|c|c|c|c|}
\hline $60-69$ & 3 & 2.8 & 22 & 21 & \multirow{5}{*}{$47.509^{\star}$} & \multirow{5}{*}{$<0.001^{\star}$} & 25 & 11.8 \\
\hline 70-79 & 14 & 13.2 & 42 & 40 & & & 56 & 26.5 \\
\hline $80-89$ & 41 & 38.7 & 21 & 20 & & & 62 & 29.4 \\
\hline $90-99$ & 29 & 27.4 & 15 & 14.3 & & & 44 & 20.9 \\
\hline$\geq 100$ & 19 & 17.9 & 5 & 4.8 & & & 24 & 11.4 \\
\hline \multicolumn{9}{|l|}{ Age } \\
\hline $21-23$ & 12 & 11.3 & 26 & 24.8 & \multirow{6}{*}{8.583} & \multirow{6}{*}{0.127} & 38 & 18 \\
\hline $24-26$ & 57 & 53.8 & 52 & 49.5 & & & 109 & 51.7 \\
\hline $27-29$ & 24 & 22.6 & 21 & 20 & & & 45 & 21.3 \\
\hline 30-31 & 6 & 5.7 & 3 & 2.9 & & & 9 & 4.3 \\
\hline $32-33$ & 3 & 2.8 & 2 & 1.9 & & & 5 & 2.4 \\
\hline$>33$ & 4 & 3.8 & 1 & 1 & & & 5 & 2.4 \\
\hline \multicolumn{9}{|c|}{ Tobacco Use } \\
\hline Yes & 7 & 6.7 & 3 & 2.9 & \multirow{2}{*}{1.68} & \multirow{2}{*}{0.195} & 10 & 4.8 \\
\hline No & 98 & 93.3 & 102 & 97.1 & & & 200 & 95.2 \\
\hline \multicolumn{9}{|c|}{ Alcohol Use } \\
\hline Yes & 2 & 1.9 & 5 & 4.8 & \multirow{2}{*}{1.33} & \multirow{2}{*}{0.249} & 7 & 3.3 \\
\hline No & 103 & 98.1 & 100 & 95.2 & & & 203 & 96.7 \\
\hline \multicolumn{9}{|c|}{ Soft Drinks } \\
\hline Yes & 52 & 49.5 & 36 & 34.3 & \multirow{2}{*}{$5.007^{\star}$} & \multirow{2}{*}{$0.025^{\star}$} & 88 & 41.9 \\
\hline No & 53 & 50.5 & 69 & 65.7 & & & 122 & 58.1 \\
\hline \multicolumn{9}{|c|}{ Fast Food } \\
\hline Yes & 63 & 60.6 & 44 & 41.9 & \multirow{2}{*}{$7.291^{*}$} & \multirow{2}{*}{$0.007^{*}$} & 107 & 51.2 \\
\hline No & 41 & 39.4 & 61 & 58.1 & & & 102 & 48.8 \\
\hline \multicolumn{9}{|c|}{ High Glycemic } \\
\hline Yes & 67 & 63.8 & 67 & 63.8 & \multirow{2}{*}{0} & \multirow{2}{*}{1} & 134 & 63.8 \\
\hline No & 38 & 36.2 & 38 & 36.2 & & & 76 & 36.2 \\
\hline \multicolumn{9}{|c|}{ Red Meats } \\
\hline Yes & 74 & 70.5 & 43 & 41 & \multirow{2}{*}{$18.547^{*}$} & \multirow{2}{*}{$<0.001^{*}$} & 117 & 55.7 \\
\hline No & 31 & 29.5 & 62 & 59 & & & 93 & 44.3 \\
\hline \multicolumn{9}{|c|}{ Aerobic Exercise } \\
\hline Yes & 60 & 57.1 & 43 & 41.3 & \multirow{2}{*}{$5.216^{\star}$} & & 103 & 49.3 \\
\hline No & 45 & 42.9 & 61 & 58.7 & & & 106 & 50.7 \\
\hline Amount & & & & & & & & \\
\hline$<6$ & 25 & 23.6 & 29 & 27.6 & & & 54 & 25.6 \\
\hline 6 to 8 & 76 & 71.7 & 72 & 68.6 & 0.511 & 0.775 & 148 & 70.1 \\
\hline$>8$ & 5 & 4.7 & 4 & 3.8 & & & 9 & 4.3 \\
\hline Major D & & & & & & & & \\
\hline Yes & 17 & 16 & 16 & 15.2 & & & 33 & 15.6 \\
\hline No & 89 & 84 & 89 & 84.8 & & & 178 & 84.4 \\
\hline
\end{tabular}




\section{Cureus}

Feeling Depressed

\begin{tabular}{|c|c|c|c|c|c|c|c|c|}
\hline Yes & 22 & 20.8 & 29 & 27.6 & \multirow{2}{*}{1.356} & \multirow{2}{*}{0.244} & 51 & 24.2 \\
\hline No & 84 & 79.2 & 76 & 72.4 & & & 160 & 75.8 \\
\hline \multicolumn{9}{|c|}{ Loss of Pleasure } \\
\hline Yes & 23 & 21.7 & 18 & 17.1 & \multirow{2}{*}{0.699} & \multirow{2}{*}{0.403} & 41 & 19.4 \\
\hline No & 83 & 78.3 & 87 & 82.9 & & & 170 & 80.6 \\
\hline \multicolumn{9}{|c|}{ Generalized Anxiety Disorder } \\
\hline Yes & 15 & 14.2 & 30 & 28.6 & \multirow{2}{*}{$6.538^{*}$} & \multirow{2}{*}{$.011^{\star}$} & 45 & 21.3 \\
\hline No & 91 & 85.8 & 75 & 71.4 & & & 166 & 78.7 \\
\hline \multicolumn{9}{|c|}{ Uncontrolled Anxiety } \\
\hline Yes & 63 & 59.4 & 72 & 68.6 & \multirow{2}{*}{1.911} & \multirow{2}{*}{0.167} & 135 & 64 \\
\hline No & 43 & 40.6 & 33 & 31.4 & & & 76 & 36 \\
\hline \multicolumn{9}{|c|}{ Worrying } \\
\hline Yes & 16 & 15.1 & 32 & 30.5 & \multirow{2}{*}{$7.102^{*}$} & \multirow{2}{*}{$0.008^{*}$} & 48 & 22.7 \\
\hline No & 90 & 84.9 & 73 & 69.5 & & & 163 & 77.3 \\
\hline \multicolumn{9}{|c|}{ Social Support } \\
\hline Yes & 97 & 91.5 & 100 & 95.2 & \multirow{2}{*}{1.184} & \multirow{2}{*}{0.277} & 197 & 93.4 \\
\hline No & 9 & 8.5 & 5 & 4.8 & & & 14 & 6.6 \\
\hline \multicolumn{9}{|c|}{ Family History: Cardiovascular Event } \\
\hline Yes & 43 & 41 & 61 & 58.1 & \multirow{2}{*}{$6.172^{*}$} & \multirow{2}{*}{$0.013^{*}$} & 104 & 49.5 \\
\hline No & 62 & 59 & 44 & 41.9 & & & 106 & 50.5 \\
\hline \multicolumn{9}{|c|}{ Family History: Type 2 Diabetes } \\
\hline Yes & 36 & 34.3 & 52 & 49.5 & \multirow{2}{*}{$5.007^{\star}$} & \multirow{2}{*}{$0.025^{\star}$} & 88 & 41.9 \\
\hline No & 69 & 65.7 & 53 & 50.5 & & & 122 & 58.1 \\
\hline
\end{tabular}

\section{TABLE 4: Distribution of blood pressure categories and risk factors in males and females}

A total of 106 males and 105 females were surveyed $(n=211)$, where 9 students did not answer at least one survey question. Pearson Chi-square was performed for each risk factor. ${ }^{*} p<0.05$

\section{Discussion}

We studied the prevalence of 2017 ACC/AHA hypertension categories and associated risk factors in first- and second-year medical students at Lincoln Memorial University - DeBusk College of Osteopathic Medicine. We found that about two-thirds of the sample had hypertensive readings and that $17.8 \%(38 / 213)$ of students had readings that fit the criteria of stage 2 HTN. This is concerning given that the prevalence of stage 2 HTN in adults of 18-39 years of age in the United States is estimated to be about 7.5\% by the CDC 2015-2016 report [1]. Hypertension was once thought of as a chronic disease associated with the elderly population; however, it is now apparent that incidence among young adults (18-30 years of age) is becoming an issue [13]. The etiology of the increased prevalence is not completely understood; it is known that certain risk factors and diseases such as decreased physical activity, diabetes, depressive states, and central obesity are becoming more prevalent in young adults [13-15]. Medical students may be a subset of the young adult population at an increased risk of developing hypertension due to risk factors that are known to already affect this population.

In this study, we found that students who sleep less than six hours per night are at significant risk of developing elevated, stage 1 HTN, and stage 2 HTN. Studies have shown that inadequate sleep and poor sleep quality are associated with increased blood pressure [16-17]. In our study, 25.5\% (54/212) of students sleep an average of 6 hours or less per night, and we believe that the issue of sleep deprivation is prevalent 
in other medical schools. In recent work looking at medical students at SUNY Downstate Medical School, researchers found that $84.7 \%$ of students slept $<7$ hours a day during the week of an examination [18]. The study concluded that a majority of SUNY medical students are sleeping an inadequate amount during their clinical and pre-clinical years. Another study at a large U.S. public university found that over one-third of students sleep less than 7 hours and about the same proportion pull at least one all-nighter in an academic year [19]. Although the lack of sleep may be contributing to hypertension in our study, we do not believe it is the only cause of the higher prevalence of HTN. The CDC determined that the percent of American adults sleeping less than 6 hours per night ranged from $27.7 \%$ to $39.8 \%$ [20]. Our study found that $25.5 \%$ of medical students are sleeping less than 6 hours per night. If lack of sleep were to play a major role in causing HTN in our sample, we would expect a greater percentage of students sleeping less than 6 hours per night in comparison to that of the CDC's data.

This study also found that an increase in waist circumference was significantly predictive of developing stage $1 \mathrm{HTN}$ and stage $2 \mathrm{HTN}$. It is known that waist circumference is a strong independent predictor of hypertension [21]. Males with waist size $\geqslant 102 \mathrm{~cm}$ have a 3.04 times greater probability of being hypertensive than males with waist sizes $<94 \mathrm{~cm}$; females with waist sizes $\geqslant 88 \mathrm{~cm}$ have a two-fold increased risk for hypertension than females with waist sizes $<80 \mathrm{~cm}$ [22]. In our sample, about $18.9 \%(20 / 106)$ of males had a waist circumference of $100 \mathrm{~cm}$ or greater, and 18.1\% (19/105) of females had a waist circumference of $90 \mathrm{~cm}$ or greater. This means that about one fifth (39/211) of male and female students have waist circumferences that increase their likelihood of developing hypertension based on the previously discussed study. Lifestyle factors such as diet and exercise are known to be major variables that influence waist circumference. In our sample, 51.0\% (107/210) of students eat fast food at least once a week, and only $49 \%(103 / 210)$ of students engage in an aerobic activity (such as brisk walk) three to four times a week averaging 40 minutes a session. The proportion of students in the sample who consume red meats, high glycemic foods, and soft drinks are similar to that of fast food.

Finally, being male was a significant predictor of elevated and stage 2 HTN. This finding parallels past research using the National Longitudinal Study of Adolescent to Adult that found among the US young adults, women are far less likely to be hypertensive than men (12\% vs $27 \%$ ) [23]. The same study also suggests that hypertension awareness among young men and women is low, but is especially lower in men. In our sample $63.3 \%$ (135/213) had hypertensive readings; however, only $2.3 \%(5 / 212)$ have been diagnosed with hypertension. This discrepancy begs the question if medical students are a subpopulation of young adults that are also unaware of their own blood pressure. In our univariate analysis of risk factors in males and females, we found that men had higher waist circumferences. In addition, a greater proportion of men consumed more soft drinks, fast food, and red meats than women. It could be the case that the relatively poor diet in this sample of male medical students is causing the disparity in the prevalence of hypertension between females and males.

No statistically significant difference was found in the proportion of students positive for generalized anxiety disorder risk, major depressive disorder risk, feelings of depression, and anhedonia in different blood pressure groups (Table 1). Likewise, binomial logistic regression did not show the previous mental health variables to be predictive of hypertension (Table 2). However, it is worth mentioning that $15.6 \%$ $(33 / 212)$ of the sample are at risk for major depressive disorder and $21.2 \%(45 / 212)$ are at risk for generalized anxiety disorder. The prevalence of MDD in American adults seems to decline with age and is estimated to be $13.1 \%$ in adults between $18-25$ years of age; $7.7 \%$ for adults $26-49$ years of age; and $4.7 \%$ for those 50 years of age and greater according to the CDC [24]. Interestingly, our sample shows the inverse with a prevalence of $10.7 \%(12 / 112)$ in students of $21-25$ years of age and $21 \%(21 / 100)$ in students between $26-37$ years of age. In terms of GAD risk in our study, there is a prevalence of 22.3\% (25/112) in students of 21-25 years of age, and $20 \%$ (20/100) in students of 26-37 years of age. According to the national comorbidity survey, an estimated $2.7 \%$ of American adults experience GAD at some point during a calendar year [25].

Literature suggests that as a student progresses through medical school, mental health declines [26]. Prevalence estimates of major depressive disorder and feelings of depression in medical students vary largely in studies, but a recent meta-analysis from 167 cross-sectional studies estimates that the prevalence of MDD and feelings of depression is $27.2 \%$ in medical students [27]. In our study, there was a significant difference between the proportion of males and females at risk for GAD. In our sample, 28.6\% (30/105) of women were at risk for GAD, while only $14.2 \%(15 / 106)$ of men were at risk $\left(X^{2}(1, N=211)=6.538, p=0.011\right)$. There was no significant difference in MDD between males and females. In terms of MDD, 15.2\% (16/105) of women were at risk, while $16.0 \%(17 / 106)$ of men were at risk $\left(X^{2}(1, N=211)=0.026, p=0.873\right)$. Previous literature has found that there is a positive association between chronic poor mental health and hypertension [28]. However, our study showed no relationship between mental health and hypertension. This discrepancy could be the result of not evaluating for chronic mental health in our sample.

Even though this study found hypertension in about two-thirds of medical students, limitations need to be considered. Although we were able to take repeated measurements of an individual's blood pressure, this is not adequate to diagnose someone with hypertension. A diagnosis should only be made after the average of two or more readings are obtained on two or more occasions [2]. In addition, our sample size is small when compared to the estimated 60,000 pre-clinical medical students in the United States [29-30]. Further, 
the study only surveyed students at one American medical school. Therefore, for these three reasons, the results of this study cannot be generalized. Further studies across various medical schools are currently being implemented to further evaluate the prevalence of HTN. In addition, this is a cross-sectional study, so we did not assess the duration that our chosen risk factors have been present in the sample. The assessment of MDD and GAD was only surveyed in the last two weeks prior to a participant completing a questionnaire. Evaluation of a past history of MDD and GAD may have shown that individuals in our sample with chronic mental health issues have statistically higher rates of hypertension. Finally, all the information obtained from medical students other than waist circumference and blood pressure was self-reported, therefore behaviors may have been over or underestimated.

\section{Conclusions}

A small group of pre-clinical medical students has more than a two times increase in prevalence of stage 2 HTN readings in comparison to Americans 18-39 years diagnosed with stage 2 HTN. The significant predictive risk factors found in this study were sleep, waist size, and gender. The influence these factor have on the wider medical student population is not fully understood. The degree to which these findings may be seen in other medical schools is the larger question postulated upon closing this study. We encourage future work at different medical schools in the United States so there can be a better understanding of the prevalence and risk factors of the of 2017 ACC/AHA hypertension categories in pre-clinical medical students.

\section{Additional Information \\ Disclosures}

Human subjects: Consent was obtained by all participants in this study. Lincoln Memorial University Institutional Review Board issued approval 756 V.0. The institutional review board of Lincoln Memorial University in Harrogate, Tennessee issued approved this study. . Animal subjects: All authors have confirmed that this study did not involve animal subjects or tissue. Conflicts of interest: In compliance with the ICMJE uniform disclosure form, all authors declare the following: Payment/services info: All authors have declared that no financial support was received from any organization for the submitted work. Financial relationships: All authors have declared that they have no financial relationships at present or within the previous three years with any organizations that might have an interest in the submitted work. Other relationships: All authors have declared that there are no other relationships or activities that could appear to have influenced the submitted work.

\section{References}

1. Fryar CD, Ostchega Y, Hales CM, Zhang G, Kruszon-Moran D: Hypertension prevalence and control among adults: United States, 2015-2016. National Center for Health Statistics. 2017, 289:Accessed: Febuary 16, 2020: https://www.cdc.gov/nchs/products/databriefs/db289.htm.

2. Whelton PK, Carey RM, Aronow WS, et al.: 2017 ACC /AHA /AAPA /ABC /ACPM /AGS/APhA/ASH/ASPC/NMA/PCNA Guideline for the Prevention, Detection, Evaluation, and Management of High Blood Pressure in Adults: Executive Summary: A Report of the American College of Cardiology/American Heart Association Task Force on Clinical Practice Guidelines. Hypertension. 2018, 71:1269-1324. Accessed: Febuary 16 2020: 10.1161/HYP.0000000000000066

3. Nyombi KV, Kizito S, Mukunya D, et al.: High prevalence of hypertension and cardiovascular disease risk factors among medical students at Makerere University College of Health Sciences, Kampala, Uganda. BMC Res Notes. 2016, 9:110. 10.1186/s13104-016-1924-7

4. Peltzer K, Pengpid S, Sychareun V, et al.: Prehypertension and psychosocial risk factors among university students in ASEAN countries. BMC Cardiovasc Disord. 2017, 17:230. 10.1186/s12872-017-0666-3

5. Abdulrahman Mazki J Alanazi, Yusef Muhana Alenezi, Tariq Hulayyil Alanazi, et al.: Prehypertension and hypertension in medical students of Northern Border University in Arar, Saudi Arabia. Egypt J Hosp Med. 2018, 70:33-38. 10.12816/0042959

6. Huang Y, Wang S, Cai X, et al.: Prehypertension and incidence of cardiovascular disease: a meta-analysis . BMC Med. 2013, 11:177-2013. 10.1186/1741-7015-11-177

7. Jee SH, He J, Whelton PK, Suh I, Klag MJ: The effect of chronic coffee drinking on blood pressure: a metaanalysis of controlled clinical trials. Hypertension. 1999, 33:647-652. 10.1161/01.hyp.33.2.647

8. Noordzij M, Uiterwaal CS, Arends LR, Kok FJ, Grobbee DE, Geleijnse JM: Blood pressure response to chronic intake of coffee and caffeine: a meta-analysis of randomized controlled trials. J Hypertens. 2005, 23:921928. 10.1097/01.hjh.0000166828.94699.1d

9. Kroenke K, Spitzer RL, Williams JB: The patient health questionnaire-2: validity of a two-item depression screener. Med Care. 2003, 41:1284. 10.1097/01.MLR.0000093487.78664.3C

10. Kroenke K, Spitzer RL, Williams JB, Monahan PO, Löwe B: Anxiety disorders in primary care: prevalence, impairment, comorbidity, and detection. Ann Intern Med. 2007, 146:317-325. 10.7326/0003-4819-146-5200703060-00004

11. Muntner P, Shimbo D, Carey RM, et al.: Measurement of blood pressure in humans: a scientific statement from the American Heart Association. Hypertension. 2019, 73:35-66. 10.1161/HYP.0000000000000087

12. Cornier MA, Després JP, Davis N, et al.: Physical Activity and Metabolism; Council on Arteriosclerosis; Thrombosis and Vascular Biology; Council on Cardiovascular Disease in the Young; Council on Cardiovascular Radiology and Intervention; Council on Cardiovascular Nursing, Council on Epidemiology and Prevention; Council on the Kidney in Cardiovascular Disease, and Stroke Council. Assessing adiposity: a 
scientific statement from the American Heart Association. Circulation. 2011, 124:1996-2019. 10.1161/CIR.0b013e318233bc6a

13. De Venecia T, Lu M, Figueredo VM: Hypertension in young adults. Postgrad Med. 2016, 128:201-207. 10.1080/00325481.2016.1147927

14. Yan LL, Liu K, Matthews KA, et al.: Psychosocial factors and risk of hypertension. The Coronary Artery Risk Development in Young Adults (CARDIA) study. JAMA. 2003, 290:2138-2148. 10.1001/jama.290.16.2138

15. Davidson K, Jonas BS, Dixon KE, et al.: Do depression symptoms predict early hypertension incidence in young adults in the CARDIA study?. Arch Intern Med. 2000, 160(10):1495-1500. 10.1001/archinte.160.10.1495

16. Aggarwal B, Makarem N, Shah R, Emin M, Wei Y, St-Onge MP, Jelic S: Effects of inadequate sleep on blood pressure and endothelial inflammation in women: findings from the American Heart Association Go Red for Women Strategically Focused Research Network. J Am Heart Assoc. 2018, 9:e008590. 10.1161/JAHA.118.008590

17. Hwang H, Lee J, Lee S, et al.: The relationship between hypertension and sleep duration: an analysis of the fifth Korea National Health and Nutrition Examination Survey (KNHANES V 3). Clin Hypertens. 2015, 21:8. 10.1186/s40885-015-0020-y

18. Ahmed N, Sadat M, Cukor D: Sleep knowledge and behaviors in medical students: results of a single center survey. Acad Psychiatry. 2017, 41:674-678. 10.1007/s40596-016-0655-3

19. Johnson KM, Simon N, Wicks M, Barr K, O’Connor K, Schaad D: Amount of sleep, daytime sleepiness, hazardous driving, and quality of life of second year medical students. Acad Psychiatry. 2017, 41:669-673. 10.1007/s40596-017-0668-6

20. QuickStats: Percentage of Adults Who Average $\leqslant 6$ Hours of Sleep, * by Family Income Group $\dagger$ and Metropolitan Status of Residence§ - National Health Interview Survey, United States, 2013. (2015). Accessed: Febuary 16, 2020: https://www.cdc.gov/mmwr/preview/mmwrhtml/mm6412a10.htm.

21. Siani A, Cappuccio FP, Barba G, et al.: The relationship of waist circumference toblood pressure: the Olivetti Heart Study. Am J Hypertens. 2002, 15:780-786. 10.1016/s0895-7061(02)02976-X

22. Guagnano M, Ballone E, Colagrande V, et al.: Large waist circumference and risk of hypertension . Int J Obes. 2001, 25:1360-1364. 10.1038/sj.ijo.0801722

23. Everett B, Zajacova A: Gender differences in hypertension and hypertension awareness among young adults . Biodemography Soc Biol. 2015, 61:1-17. 10.1080/19485565.2014.929488

24. 2017 National Survey on Drug Use and Health: Methodological Summary and Definitions . (2018). Accessed: Febuary 16, 2020: https://www.samhsa.gov/data/sites/default/files/cbhsqreports/NSDUHMethodSummDefs2017/NSDUHMethodSummDefs2017.htm.

25. National Comorbidity Survey (NCS). (2007). Accessed: Feburary 16, 2020 : https://www.hcp.med.harvard.edu/ncs/index.php.

26. Dyrbye LN, Thomas MR, Shanafelt TD: Medical student distress: causes, consequences, and proposed solutions. Mayo Clin Proc. 2005, 80:1613-1622. 10.4065/80.12.1613

27. Rotenstein LS, Ramos MA, Torre M, et al.: Prevalence of depression, depressive symptoms, and suicidal ideation among medical students: a systematic review and meta-analysis. JAMA. 2016, 316:2214-2236. 10.1001/jama.2016.17324

28. Stein DJ, Aguilar-Gaxiola S, Alonso J, et al.: Associations between mental disorders and subsequent onset of hypertension. Gen Hosp Psychiatry. 2014, 36:142-149. 10.1016/j.genhosppsych.2013.11.002

29. American Association of Colleges of Osteopathic Medicine. Osteopathic Medical Colleges Matriculants by U.S. States and Territories. (2018). Accessed: Feburary 16, 2020: https://www.aacom.org/docs/defaultsource/data-and trends/2018matriculantsbystatebycom.pdf?sfvrsn=85b63597_6\&fbclid=I....

30. Association of American Colleges. (2017). Accessed: Feburary 16, 2020: https://www.aamc.org/system/files/d/1/5-2017_applicant_and_matriculant_data_tables.pdf. 